\title{
ECONOMÍA SUMERGIDA Y RELACIONES LABORALES EN EUROPA*
}

\author{
SHADOW ECONOMY AND EUROPEAN LABOUR \\ RELATIONS \\ L'ÉCONOMIE SOUTERRAINE ET LES RELATIONS \\ INDUSTRIELLES EN EUROPE
}

Jordi GARCÍA VIÑA**

\begin{abstract}
RESUMEN: La economía sumergida no es un fenómeno nuevo en las relaciones laborales, ni se limita a un país o zona determinada. Es una situación que afecta a la mayoría de los países y que se está viendo incrementada con la crisis económica. Los efectos negativos de la economía sumergida son múltiples y afectan a todos los sujetos económicos: Estados, empresas y trabajadores. Por esta razón, hay que buscar soluciones para erradicar o limitar sus consecuencias, aunque no es fácil determinar cuáles pueden ser.
\end{abstract}

Palabras clave: Economía sumergida, relaciones laborales, empleo, fraude.

ABSTRACT: The shadow economy is not a new phenomenon in labour relations; nor is it limited to one country or area. It is a situation that affects most of the countries and that is getting worse with the economic crisis. The negative effects of the shadow economy are multiple and they affect all the economic actors: governments, companies and

* Recibido el 18 de noviembre de 2014 y aceptado para su publicación el 23 de marzo de 2015.

El origen de este trabajo es su presentación en el seminario "Las nuevas condiciones del trabajo en el contexto de la globalización económica. ¿Hacia un nuevo derecho del trabajo?”, Proyecto Conacyt, núm. 169282, celebrado los días 21 y 22 de octubre en el Instituto de Investigaciones Económicas de la UNAM, dirigido por el doctor José Alfonso Bouzas Ortíz, al que agradezco su confianza y gentileza.

** Catedrático de Derecho del trabajo y de la seguridad social, Universidad de Barcelona, España(jordi.garcia@ub.edu). 
workers. For this reason, solutions must be found to eradicate or limit its consequences, despite the fact that it is not easy to determine what these consecuences may be.

Key Words: Shadow economy, labour relations, employment, fraud.

RÉSUMÉ: L'économie souterraine n'est pas un nouveau phénomène au sein des relations industrielles. De plus, elle ne se limite pas à un pays où à une région spécifique. Il s'agit d'une situation qui touche la majorité des pays et qui augmente avec la crise économique. Les effets négatifs de l'économie souterraine sont multiples et touchent tous les acteurs économiques: les États, les entreprises et les travailleurs. C'est pour cette raison qu'il est nécessaire de trouver des solutions pour éradiquer ou restreindre ses conséquences, bien qu'il ne soit pas simple de les identifier.

Mots-clés: Économie souterraine, relations industrielles, emploi, fraude.

SUMARIO: I. Introducción. II. La crisis económica de 2007. III. La economía sumergida y el empleo irregular. IV. Medidas para reducir la economía sumergida. V. Conclusiones. VI. Bibliografía.

\section{INTRODUCCIÓN}

a crisis económica generalizada que sufre principalmente Europa desde el desplome del precio del petróleo ha supuesto una modificación radical en cuanto a los planteamientos del derecho del trabajo y de la seguridad social, agravada por la interferencia de la denominada "economía sumergida".

En la Europa de 1945-1973 se establece el sistema de empleo llamado "fordismo", cuya relación laboral presenta las siguientes notas principales:

- La contratación será de duración indeterminada o, dicho de otra manera, la mayoría de todos los contratos son indefinidos. En España es curioso que la Ley de Contrato de Trabajo de 1944 no se expresaba en favor de la indefinición y que fueron las reglamentaciones nacionales del trabajo, junto a la doctrina instaurada por el Tribunal Supremo, las que permitieron este cambio. 
- Todos estos contratos indefinidos eran celebrados a tiempo completo, de manera que el tiempo parcial quedaba relegada a supuestos muy concretos.

- Esta prestación se realizaba, casi durante toda la vida del trabajador, por cuenta de un empleador único.

- Existía una importante protección contra el despido injustificado.

- Desarrollo del Estado del bienestar, entendido como la máxima preocupación por parte de los Estados hacia la protección social de los trabajadores y usuarios del sistema público de seguridad social.

El conflicto árabe-israelí de 1973 originó una situación económica recesiva, que si bien en apariencia no era nada más que una coyuntura adversa, pronto se reveló como un estado de crisis con evidentes rasgos de permanencia, al asociarse a ella la incorporación al sistema productivo de importantes cambios tecnológicos. Por tanto, esta "crisis del petróleo" supuso una crisis estructural, de la que se pueden destacar los siguientes factores económicos: competencia internacional; innovación técnica; modificaciones radicales en la organización de la producción; nuevos métodos de gestión de la mano de obra; evolución de las aspiraciones y necesidades de los trabajadores, entre muchas otras.

Esta metamorfosis significó un debate sobre la flexibilidad en el empleo, que puso en tela de juicio este modelo de relación clásico. Las notas principales de la crisis de este modelo fueron las siguientes:

- Multiplicación de los contratos laborales de duración determinada.

- Normalización del trabajo a tiempo parcial.

- Externalización de la producción y del empleo.

- Aparición de economía clandestina o sumergida.

Si se lleva a cabo un breve análisis de cada nota, se puede observar que aparece una multiplicación de las modalidades de contratación por tiempo determinado, especialmente en aquellos países donde el despido es causal y de coste muy elevado. Respecto al trabajo a tiempo parcial, ha tenido un gran desarrollo, porque favorece la flexibilidad de las empresas. Así pues, aparecen las subcontrataciones con otras empresas para todas aquellas actividades de servicio, además de la figura de las empresas de empleo temporal. Por último, se impone la economía sumergida como medio de reducir los costes labora- 
les. En esta situación se produce una oposición crítica a la situación anterior por entender que engendraba rigideces incompatibles con las necesidades de las empresas, que necesitaban adaptarse a un entorno económico cambiante y competitivo.

Además, cabe añadir la internacionalización de la economía, el Acuerdo General sobre Aranceles y Comercio, el Tratado de Libre Comercio, la ascensión en el ranking de competitividad de los países del sureste asiático o los profundos cambios estructurales en cuanto a la organización de las empresas, concretamente el just in time.

La clave para la solución de la crisis fue la búsqueda de la flexibilidad en las relaciones laborales. Este concepto, de naturaleza extremadamente amplia, presenta lectura positivas y negativas, según sea el sujeto que al que se le aplica. Este proceso puede observarse claramente desde tres puntos de vista:

- Desrregulación del mercado de trabajo. Pretende la búsqueda de una mejora de los niveles de empleo, justificando el objetivo del crecimiento del empleo sobre la estabilidad en el mismo y la defensa de la competitividad empresarial a costa de una cierta pérdida de beneficios sociales por parte de los trabajadores.

- Desrregulación de las condiciones de trabajo en favor de la negociación colectiva. Se potencia por medio de la flexibilización de los requisitos de negociación y la admisión de los pactos colectivos. Además, se limita la intervención de la administración pública en la regulación de las condiciones de trabajo.

- Reducción del gasto del sistema de la seguridad social; por ejemplo, respecto a las prestaciones de desempleo, incapacidad temporal, jubilación, o en la exclusión o reducción de la cobertura de ciertos colectivos.

\section{LA CRISIS ECONÓMICA DE 2007}

Esta situación de replanteamiento de los principios del derecho laboral vuelve a estar presente en la actualidad. De hecho, la crisis financiera iniciada en el verano de 2007 en Estados Unidos y la posterior recesión económica a escala mundial supusieron el inicio de una crisis económica y de empleo de enormes proporciones, que difícilmente permite anunciar su salida. 
En la Unión Europea, el número de personas desempleadas ha aumentado de manera vertiginosa por este motivo. Aunque este incremento afecta a todos los Estados miembros y a todos los colectivos, ya sean hombres o mujeres y/o jóvenes o personas mayores, el impacto ha sido muy diverso, y en esta situación pueden influir diversos factores, como las estructuras productivas e institucionales de cada país, así como las medidas económicas y de empleo puestas en práctica.

Conforme a las estadísticas de Eurostat, el número de personas desempleadas se ha incrementado mensualmente desde marzo de 2008, donde se observan los porcentajes más bajos. España e Italia fueron los primeros países donde el desempleo empezó a incrementarse (aproximadamente a partir de mayo de 2007), seguidos de Irlanda, Luxemburgo, Letonia y Lituania (segunda mitad del mismo año), y Francia (principios del año siguiente).

Desde mayo de 2007, el número de personas desempleadas en los países que tienen implantado el euro se ha incrementado en 3.7 millones hasta alcanzar los 15 millones, mientras que en el mismo periodo, si se tiene en cuenta a todos los países que forman la Unión Europea, el desempleo ha subido en 5.4 millones hasta superar los 21.5 millones. ${ }^{1}$

\section{Gráfica 1 \\ DESEMPLEO}

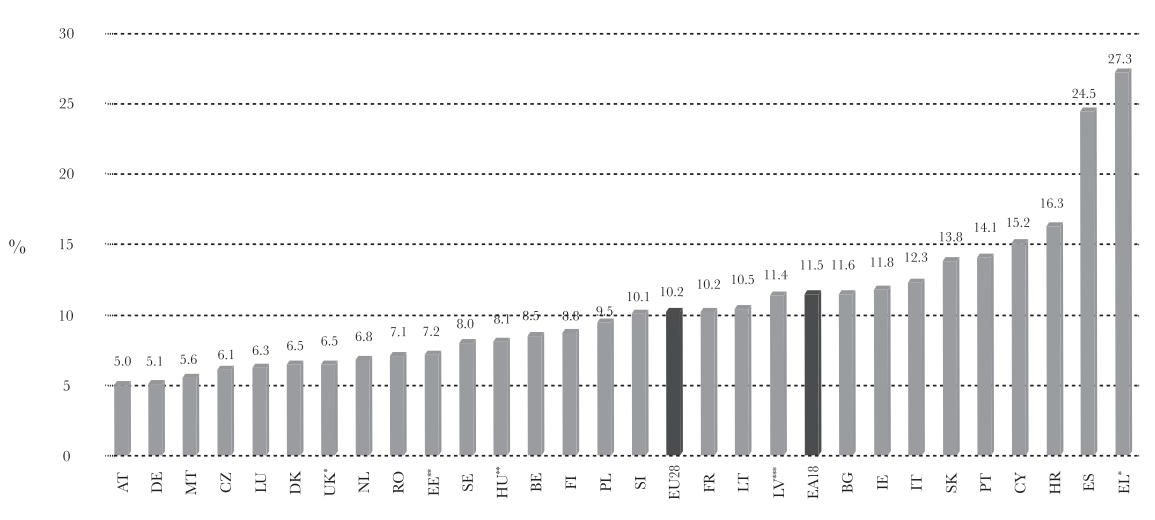

*April $2014{ }^{* *}$ May $2014{ }^{* * *}$ QI 2014

Fuente: Eurostat.

1 http://epp.eurostat.ec.europa.eu/statistics_explained/index.php/Unemployment_statistics. 
No sólo hay que tener en cuenta el momento de arranque de la situación de desempleo, sino también la intensidad, que es muy diferente si se comparan a los distintos países. En la mayoría de países, el incremento medio mensual ha sido del $0.1 \%$ al $0.2 \%$; sin embargo, estos incrementos han sido mucho mayores en los países bálticos y en España. ${ }^{2}$

Por otra parte, el desempleo no ha afectado por igual a todos los trabajadores: el peso de la crisis ha recaído principalmente en los trabajadores jóvenes; el desempleo masculino ha evolucionado de forma más negativa que el femenino, y, por último, los ajustes del empleo ante la crisis han sido mucho mayores entre los trabajadores con contratos temporales y/o niveles formativos más bajos. ${ }^{3}$

\section{Gráfica 2}

DESEMPLEO JUVENIL

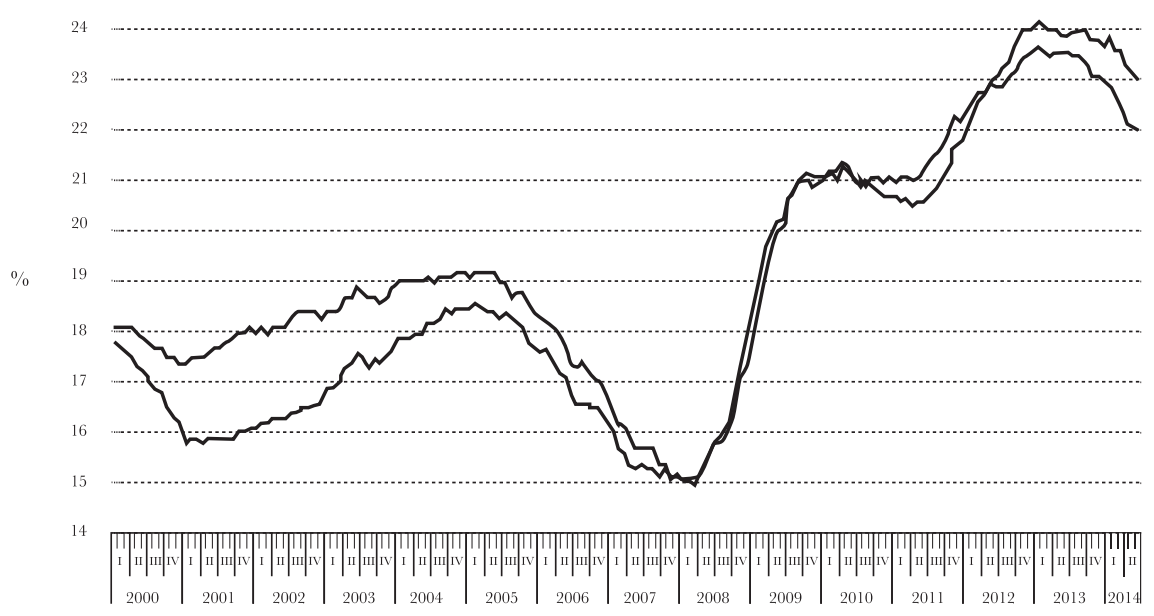

Fuente: Eurostat.

2 Eurostat, "Sharp incrase in unemployment in the EU", Statistics in focus, 53/2009.

3 Eurostat, "The impact of the crisis on employment", Statitics in focus, 79/2009. 
En este contexto, aparecen dos incógnitas de difícil resolución: hasta cuándo va a durar la crisis económica, y qué incidencia va a tener la recuperación de la crisis con el desempleo.

La Organización para la Cooperación y el Desarrollo Económico estima que el impacto de la crisis en el empleo se va a prolongar más allá del inicio de la recuperación económica. El aumento del paro de larga duración, así como la pérdida de capital humano a través de la descualificación de los parados de larga duración, hará más difícil la recuperación de los niveles de empleo anteriores a la situación de la crisis, por lo que éstos pueden tardar mucho tiempo en reponerse o pueden, incluso, no volver a alcanzarse. ${ }^{4}$

De la misma manera, la Comisión Europea espera un empeoramiento de las condiciones del mercado de trabajo durante todavía algún tiempo después de que comience a producirse la reactivación del crecimiento económico, por lo que estima que la Unión Europea puede alcanzar durante 2014 los 27 millones de residentes de la Unión Europea de paro. ${ }^{5}$

\section{LA ECONOMÍA SUMERGIDA Y EL EMPLEO IRREGULAR}

Mientras Europa está intentando salir del terrible drama de la crisis económica, con su resultado más trágico que es el desempleo, surge un elemento que complica, aún más, si cabe, esta situación. Así, la economía sumergida alcanzó a finales de 2013 un valor total estimado de 195,600 millones de euros, lo que supone un 18.6\% del PIB, ligeramente inferior al de 201,300 millones de euros (un 19\% del PIB) contabilizado al cierre de 2012. ${ }^{6}$

La situación de España se compara mal con la de otros países europeos. El último informe de Schneider señala que en países como Francia y Alemania la economía sumergida alcanza de media el 15-16\% del PIB, mientras Suecia y los países escandinavos se sitúan entre el 17.4\% y el 18.5\%. Italia se sitúa en el $26.9 \%$, frente al $22.8 \%$ de España y el $20.3 \%$ de la media de la OCDE. ${ }^{7}$

\footnotetext{
4 OECD, OECD Employment Outlook 2014, OECD Publishing, 2014.

5 http:// europa.eu/pol/pdf/flipbook/es/employment_es.pdf.

6 Jiménez Fernández, A. y Martínez Pardo-del Valle, R., La economía sumergida en España, documentos de trabajo, núm. 4, Madrid, Fundación de Estudios Financieros, 2013.

7 Schneider, F. y Johannes Kepler, J., "The Shadow Economy in Europe, 2013", Informe ATKearney/Visa, 2013.
} 
Asimismo, la evolución temporal no es clara. La economía sumergida ha caído desde 2007, año de comienzo de la crisis, tanto en Europa como en la OCDE. Se aprecia un aumento en España, ya que en 2009 la economía sumergida representaba el 23.3\%, mientras que en 2013 admitía una subida hasta el 24.6\%. Esta diferencia, en cualquier caso, no son 60,000 millones de euros.

Es cierto que el fraude del IVA y de la seguridad social se ha incrementado, pero no es menos cierto que el origen de gran parte de la economía sumergida antes de la crisis estaba relacionada con el sector inmobiliario, y éste ha caído drásticamente.

Estos datos sitúan a España, junto con Portugal, en el tercer puesto entre los países de Europa Occidental cuya economía sumergida equivale a un porcentaje mayor respecto al PIB. Por volumen total de economía sumergida en euros, España sería el quinto país del ranking, por detrás de Alemania, Italia, Francia y el Reino Unido.

La economía sumergida tiene un gran tamaño en España, que supera a las de la mayoría de los países de Europa Occidental, aunque se mantiene en un nivel similar al de la media europea (18.5\%), un total de 2.15 billones de euros. Además, el peso de la economía sumergida en España no ha cambiado sustancialmente respecto a los datos de 2008, y se concentra especialmente en los sectores de la construcción, industria y servicios.

El problema de la economía sumergida no es nuevo, ni en España ni en los países de la Unión Europea, ya que siempre ha existido una preocupación por su existencia, falta de control, tamaño y evolución, y no cabe duda de los numerosos efectos negativos que tiene en la economía, tanto desde un punto de vista macroeconómico como microeconómico, y la incertidumbre que genera en los ciudadanos y en las empresas que sí cumplen con sus deberes laborales y de seguridad social.

$\mathrm{Al}$ margen de las razones de equidad que puedan existir, la presencia de esta economía sumergida impide tener una imagen real de la verdadera situación de la economía, cuyos efectos principales son la deformación de los principales datos generales, penalizar la capacidad recaudatoria, dificultar la consolidación fiscal, y hacer más compleja la toma de decisiones de política económica. ${ }^{8}$

8 International Labor Organization, Labour inspection and undeclared work in the EU (LAB/ ADMIN Working Document No. 29), Ginebra, 2013. 
El conocimiento de la economía sumergida ha sido tradicionalmente un tema de preocupación. ${ }^{9}$ De hecho, no se trata de una cuestión nueva, sino de una materia que ha cobrado una especial relevancia dada la situación de crisis mundial y la extraordinaria cifra que puede haber alcanzado. Según un estudio realizado en noviembre de 2011, la economía sumergida cubre el 98.2\% de los países del mundo y el $92.4 \%$ de su población, y el tamaño de la economía sumergida mundial alcanza 11.1 billones dólares o el equivalente al 18.1\% del PIB mundial. ${ }^{10}$

En España, donde la crisis está siendo especialmente virulenta, con una importante recesión, paro elevado y gran caída de los ingresos de las administraciones públicas, la sospecha de que una gran parte de la actividad económica se realiza en el ámbito de la economía no declarada, suscita mayores debates en relación con su actuación como vía para salir de esta compleja situación económica, así como para reforzar el compromiso moral de la sociedad.

También la crisis económica en España ha hecho cambiar la percepción ciudadana respecto a la economía irregular. Según un estudio del Instituto de Estudios Fiscales, en 2011 un 67\% de los ciudadanos consideraba que no se podía justificar el fraude fiscal, frente a un 51\% que opinaba igualmente en 2007. Asimismo, sólo un 25\% consideraba que hay circunstancias que la pueden justificar, frente a un 41\% que pensaba así en 2007..11

Una de las cuestiones fundamentales a la hora de analizar el fenómeno de la economía sumergida es tratar de definir dicho concepto, ya que no es único. Las definiciones existentes son bastante parecidas. Así, la Comisión Europea se refiere a la economía sumergida como aquella actividad remunerada que siendo legal en cuanto a su naturaleza, no es declarada a las autoridades públicas. ${ }^{12}$ Para la OCDE, este fenómeno comprende aquellas actividades que son productivas en sentido económico y relativamente legales (dependiendo

\footnotetext{
9 bttp://eptbinktank.eu/2013/08/13/tackling-undeclared-work/.
}

10 Tax Justice Network, "The cost of tax abuse. A briefing paper on the cost of tax evasion worldwide", 2011.

11 Instituto de Estudios Fiscales, Opiniones y actitudes fiscales de los españoles en 2011, documentos, núm. 19, Madrid, 2012.

12 Communication from the Commission to the Council, the European Parliament, the European Economic and Social Committee and the Committee of the Regions-Stepping up the fight against undeclared work /* COM/2007/0628 final. 
de los estándares y regulaciones locales), pero que son ocultadas deliberadamente a las autoridades públicas. ${ }^{13}$

Como se puede observar, en ambos casos se trata de actividades que son legales en cuanto a su naturaleza, pero que son no declaradas a las diferentes autoridades públicas.

Se suelen incluir actividades que son aceptadas socialmente, y que no pagan impuestos fiscales; por ejemplo, prestaciones de servicios por parte de trabajadores independientes que no pagan IVA o actividades sin la correspondiente retención y liquidación del IRPF; personas que perciben indebidamente prestaciones de la seguridad social; ${ }^{14}$ empresas que venden sus productos o prestan sus servicios sin cumplir los requisitos legales derivados de las normas tributarias, laborales o de seguridad social (economía informal), o personas que trabajan sin que hayan sido dados de alta a la seguridad social, que no se cotice por ellos, total o parcialmente, y/o que perciban una parte de su salario de manera "extraoficial".

En este tema hay que tener en cuenta la gran importancia que tiene la conexión entre este concepto de economía sumergida y empleo informal, en especial cuando se vincula al denominado trabajo independiente o autónomo o al empleo doméstico.

Es especialmente en estos apartados, posiblemente los más numerosos, donde aparece claramente esta teórica contradicción, ya que existe un claro consenso en reservar el concepto de economía sumergida a este tipo de actividades no declaradas.

Sin embargo, desde el momento en que se trata de prestaciones de servicios que pretenden ser ocultadas, totalmente o en parte, es evidente que se produce un incumplimiento de determinadas obligaciones legales, principalmente en lo que se refiere a deberes tributarios y de cotización a la seguridad social, por no mencionar el no cumplimiento de las cláusulas establecidas en los convenios colectivos, que deberían ser de aplicación.

Es difícil imaginar situaciones que son irregulares, sin que lleguen a ser ilegales, salvo que se acepte un concepto muy amplio de economía informal.

13 Véase el capítulo quinto, de título "Informal Employment and Promoting the Transition to a Salaried Economy", OECD Employment Outlook, 2004.

14 Williams, C. y Nadin, S., "Evaluating the Participation of the Unemployed in Undeclared Work. Evidence from a 27-nation European survey", mayo de 2012. 
Por tanto, a la hora de delimitar este concepto se debería incluir, si se pretende ser exhaustivo, tanto las situaciones de irregularidad fiscal como las situaciones no declaradas en el ámbito laboral, ${ }^{15}$ pudiendo ser obviadas, si acaso, las conductas que pueden ser calificadas como de ilícito penal.

Entonces, cuáles son las causas directas e indirectas de la existencia de la economía sumergida. Éstas son diversas, pero pueden ser resumidas en las siguientes: ${ }^{16}$

- Elevada presión fiscal.

- Percepción de que la relación entre los impuestos que se pagan y los servicios y prestaciones que se reciben no es la adecuada.

- Excesiva regulación y complejidad de las obligaciones tributarias, laborales y administrativas.

- Impunidad de los que defraudan.

- Falta de honradez o de conciencia cívica.

- Ineficacia de la lucha para combatirla.

Además, la existencia de economía no declarada en una sociedad supone una grave anomalía para su correcto funcionamiento por las siguientes razones: ${ }^{17}$

- Genera problemas de equidad, tanto por el lado del gasto como por el de los ingresos públicos, ya que se generan asimetrías en la provisión de servicios y en la carga impositiva soportada, así como desde el punto de vista interregional e internacional.

- Plantea problemas de eficiencia en la asignación de recursos y en la provisión de servicios por el Estado.

- Produce pérdidas recaudatorias para el sector público, que limitan las actuaciones de cualquier política estatal.

- Distorsiona la competencia empresarial al situar las actividades no declaradas al margen del pago de impuestos, de cargas laborales o de requerimientos burocráticos y administrativos.

15 Jiménez Fernández, A. y Martínez Pardo-del Valle, R., op. cit., p. 13.

16 Schneider, F., "Work in the Shadow: Micro and Macro Results", International Economic Journal, vol. 28, núm. 3, 2014.

17 Arrazola, M. et al., Dos ensayos de actualidad sobre la economía española. La economía sumergida en España, Funcas, 2011. 
- Provoca desviaciones en las estadísticas económicas oficiales (PIB, renta disponible, paro, etcétera), que a su vez impiden un adecuado diagnóstico de los problemas socioeconómicos y una correcta toma de decisiones.

- Se producen problemas de desigualdad entre ciudadanos y menoscabo de los derechos de los trabajadores contratados de forma irregular.

Es evidente que, según el estudio de Schneider, si la economía sumergida en Europa es equivalente al $18.5 \%$ de la actividad económica europea, esta realidad se ha de situar en el primer plano de la actualidad, en estos días en que los Estados buscan equilibrar las cuentas públicas, la necesidad de reducir el tamaño de la economía no declarada por medio de medidas preventivas, además de un control e inspección y mayores sanciones, o concienciando a los ciudadanos de los perjuicios que su existencia causa a la sociedad.

Por un lado, casi dos tercios del volumen estimado se concentra en los cinco mayores países europeos: Alemania, Francia, Italia, Reino Unido y España, en términos absolutos. Sin embargo, en los países del este europeo su tamaño relativo, respecto al PIB, es mucho mayor. Así, en países como Bulgaria, Croacia, Lituania y Estonia el tamaño de su economía irregular se cifra en casi un $30 \%$ del PIB. Austria y Suiza tienen los niveles más bajos, del 7\% y del 8\% de su PIB, como se observa en la siguiente gráfica y tabla.

\section{GRÁFICA 3}

ECONOMÍA SUMERGIDA CON RELACIÓN AL PIB

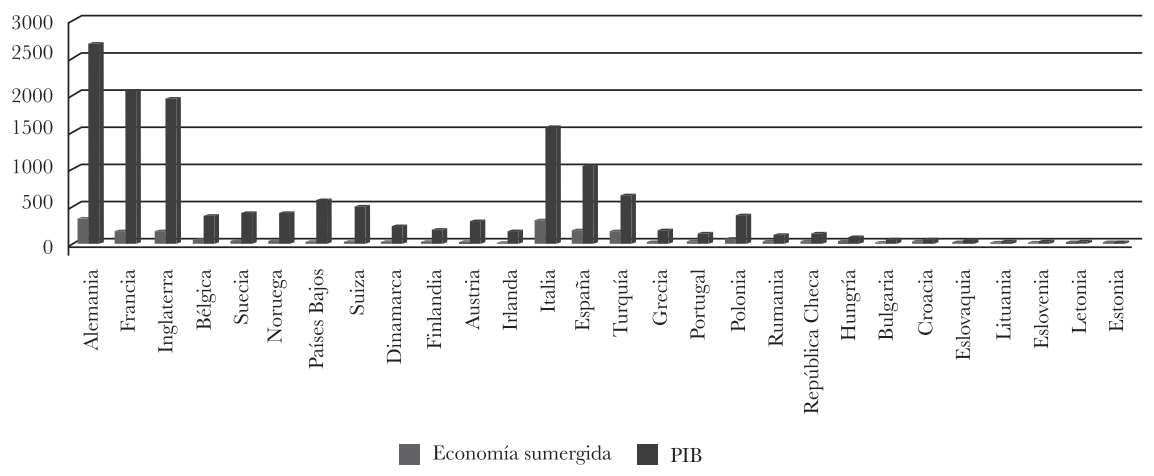

Fuente: Informe ATKearney/VISA 2013 (en millardos de euros). 
Tabla 1

ECONOMÍA SUMERGIDA CON RELACIÓN AL PIB

\begin{tabular}{|c|c|c|c|}
\hline & $\begin{array}{l}\text { Economía } \\
\text { sumergida }\end{array}$ & $P I B$ & $\begin{array}{c}\text { Economía sumergida como } \\
\text { porcentaje del PIB }\end{array}$ \\
\hline Alemania & 351 & 2,698 & $13 \%$ \\
\hline Francia & 204 & 2,062 & $10 \%$ \\
\hline Inglaterra & 189 & 1,950 & $10 \%$ \\
\hline Bélgica & 63 & 385 & $16 \%$ \\
\hline Suecia & 59 & 423 & $14 \%$ \\
\hline Noruega & 67 & 416 & $14 \%$ \\
\hline Países Bajos & 55 & 607 & $9 \%$ \\
\hline Suiza & 36 & 507 & $7 \%$ \\
\hline Dinamarca & 32 & 250 & $13 \%$ \\
\hline Finlandia & 26 & 199 & $13 \%$ \\
\hline Austria & 24 & 319 & $8 \%$ \\
\hline Irlanda & 20 & 167 & $12 \%$ \\
\hline Italia & 333 & 1,577 & $21 \%$ \\
\hline España & 196 & 1,052 & $19 \%$ \\
\hline Turquía & 177 & 667 & $26 \%$ \\
\hline Grecia & 43 & 183 & $24 \%$ \\
\hline Portugal & 31 & 164 & $19 \%$ \\
\hline Polonia & 95 & 400 & $24 \%$ \\
\hline Rumania & 40 & 139 & $28 \%$ \\
\hline República Checa & 24 & 154 & $15 \%$ \\
\hline Hungría & 23 & 103 & $22 \%$ \\
\hline Bulgaria & 13 & 41 & $31 \%$ \\
\hline Croacia & 13 & 45 & $28 \%$ \\
\hline Eslovaquia & 11 & 74 & $15 \%$ \\
\hline Lituania & 10 & 35 & $28 \%$ \\
\hline Eslovenia & 8 & 35 & $23 \%$ \\
\hline Letonia & 6 & 24 & $25 \%$ \\
\hline Estonia & 5 & 18 & $28 \%$ \\
\hline
\end{tabular}

Fuente: Informe ATKearney/VISA 2013 (en millardos de euros) 
Según los datos de este informe, el importe estimado de la economía no declarada en España asciende a 196,000 millones de euros, lo que supone un 18.6\% del PIB, similar al 18.5\% de media en el resto de los países analizados, pero muy alejado del de otros Estados europeos, como Alemania (13\%); Austria, Holanda o Suiza (por debajo del 10\% del PIB), y Francia y Reino Unido (en torno al 10\%).

Si la economía sumergida se analiza desde un punto de vista de su evolución, se puede constatar que su nivel en Europa se sitúa en la actualidad en el punto más bajo de los últimos diez años; de hecho, ha descendido desde el 22.4\% del PIB europeo en 2003 hasta el 18.5\% en 2013, y se distribuye de manera diferente entre los países de las regiones europeas, por lo que se observan claras diferencias entre norte-sur y este-oeste en lo que al tamaño de su economía sumergida se refiere.

De esta manera, hasta 2008 se había producido una disminución paulatina de la presencia de la economía sumergida en el PIB en todos los países, y que a partir de este momento, tras un repunte en 2009, se ha mantenido levemente en forma decreciente hasta los niveles actuales.

En España, según se desprende de este informe, la tendencia es la misma. Ha habido una disminución desde los niveles de 2003 (22.2\% del PIB) hasta los actuales. Comparando este comportamiento descendente con el de los países europeos más relevantes, se puede observar que si bien las curvas son similares, con la misma tendencia a lo largo del ciclo, el nivel de la economía sumergida ha sido recurrentemente superior en Italia respecto a España (en porcentaje del PIB), que a su vez ha sido muy superior a los existentes en Austria, Holanda, Francia o Reino Unido.

En cuanto a las características que determinan el volumen de la economía sumergida, hay que tener en cuenta que en momentos de crisis económica, aumento de las tasas de desempleo, disminución de los ingresos familiares, especialmente de los salarios, e incógnitas sobre la situación futura, es relativamente habitual que una de las soluciones tomadas por las personas sea recurrir a la economía sumergida. De esta manera, se consigue corregir el descenso de ingresos regulares, ya que al no tener que pagar por estas actividades los correspondientes impuestos o cotizaciones a la seguridad social, se incrementan los ingresos. 
Este comportamiento se refleja claramente en la economía europea desde el inicio de la crisis, pues el tamaño de la economía sumergida tiene una elevada correlación negativa con los ciclos económicos, como se puede observar en la siguiente gráfica.

\section{Gráfica 4}

EVOLUCIÓN DE LA ECONOMÍA SUMERGIDA EN EUROPA

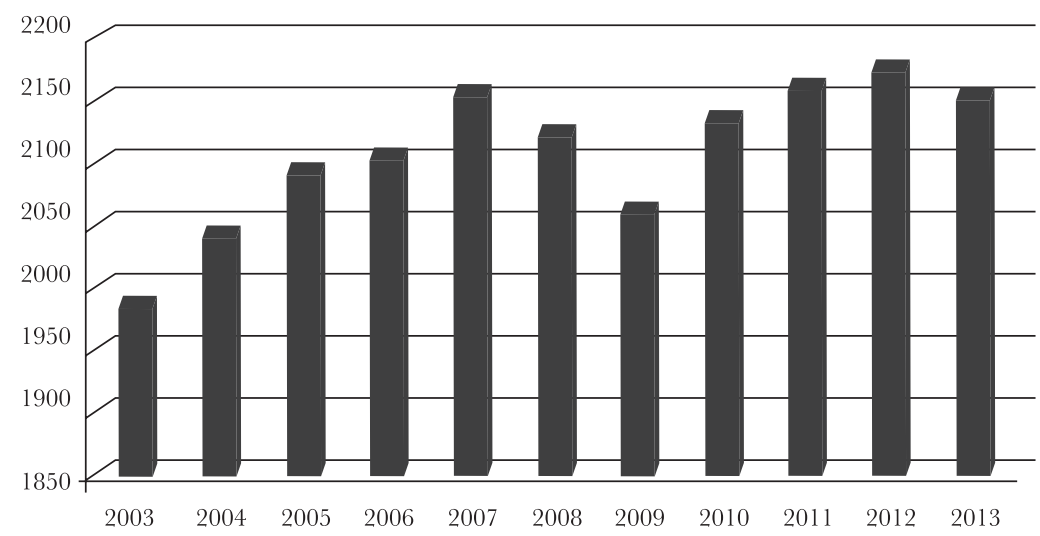

Gráfica 5

EVOLUCIÓN DE LA ECONOMÍA SUMERGIDA COMO PORCENTAJE DEL PIB

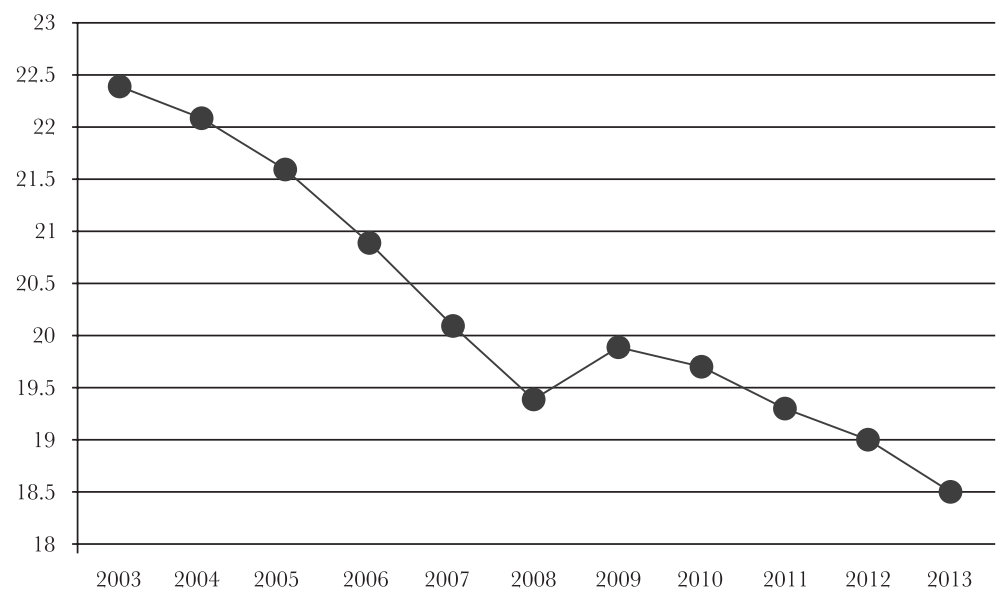

Fuente (en ambos casos): Schneider, F. y Kepler, J., Universidad de Linz, Austria; Eurostat, A. T. Kearney Analysis, 2013. 
Como se puede comprobar en estos gráficos, el volumen de economía sumergida en valores absolutos ha crecido de manera ininterrumpida entre 2003 y 2008, pero a tasas inferiores de lo que ha crecido la actividad económica "oficial":

\begin{tabular}{|c|c|}
\hline Crecimiento PIB & Crecimiento economía sumergida \\
\hline $23.4 \%$ & $7.9 \%$ \\
\hline
\end{tabular}

Este distinto porcentaje de incremento ha supuesto una reducción del peso de la economía sumergida o irregular sobre el PIB en este periodo. Así, en 2009, la economía sumergida descendió escasamente en porcentajes absolutos, pero menos de lo que ha hecho el PIB, lo que supuso un incremento de 0.5\% en términos relativos. En cambio, a partir de 2010, debido a la incipiente recuperación de la economía, los niveles de economía irregular alcanzaron situaciones previas ( 2.1 billones de euros) y se han mantenido estables desde este año. En este mismo sentido, el porcentaje sobre el PIB medio se ha situado, de manera más o menos estable, alrededor del 19\%, lo que supone una evolución parecida de la economía oficial y de la sumergida, aunque es probable que a medida que se produzca la recuperación económica, descenderá la economía sumergida.

En esta línea, un informe de Eurofound muestra una relevante correlación estadística entre los tipos de medidas seguidos por los diferentes países y el tamaño de su economía sumergida, ${ }^{18}$ cuyas conclusiones son las siguientes:

\begin{tabular}{|c|l|}
\hline 1 & $\begin{array}{l}\text { Los Estados de la UE con más carga impositiva media sobre los ingresos del } \\
\text { trabajo no tienen una mayor economía sumergida. }\end{array}$ \\
\hline 2 & $\begin{array}{l}\text { Los Estados con menor carga impositiva media sobre las rentas del trabajo } \\
\text { no están significativamente correlacionados con un menor tamaño de su } \\
\text { economía sumergida. }\end{array}$ \\
\hline 3 & $\begin{array}{l}\text { No existe evidencia de que la intervención pública en el mercado laboral } \\
\text { incremente la economía sumergida. Hay, por el contrario, una correlación } \\
\text { estadística significativa entre una mayor intervención en el mercado laboral } \\
\text { y la reducción del tamaño de la economía no declarada. }\end{array}$ \\
\hline
\end{tabular}

18 Williams, C. y Renooy, P., Tackling undeclared work in 27 European Union Member States and Norway: Approaches and measures since 2008, Dublin, Eurofound, 2013. 


\begin{tabular}{|c|l|}
\hline 4 & $\begin{array}{l}\text { Los países en los que hay un mayor gasto en protección social (en porcentaje } \\
\text { del PIB) tienen menor economía irregular. }\end{array}$ \\
\hline 5 & $\begin{array}{l}\text { Los países con mayor redistribución de la renta tienen un menor tamaño de } \\
\text { economía sumergida. }\end{array}$ \\
\hline 6 & $\begin{array}{l}\text { En los regímenes con una mayor igualdad social, el tamaño de su economía } \\
\text { sumergida es menor que en los países con mayores desigualdades sociales. }\end{array}$ \\
\hline
\end{tabular}

El informe concluye que aunque no se puede hablar de una relación causaefecto, el mayor tamaño de la economía sumergida se debe a unos menores niveles de desarrollo, de bienestar social y de regulación. Además, este informe indica que no existe correlación entre mayores tasas impositivas y mayor economía no declarada. No obstante, este informe advierte de que cualquier tipo de conclusión debe ser analizada caso por caso, en cada uno de los diferentes países. Así, en el caso de referirse a España, también hay tener en cuenta otros elementos, tales como la complejidad de la regulación, el exceso de trabas administrativas, el incremento de la carga impositiva o el tamaño de las empresas en España (en su mayoría pymes).

Una de las cuestiones a tener en cuenta a la hora de estudiar la economía sumergida consiste en determinar qué tipo de población tiene una relación más directa con esta situación o, dicho de otra manera, se beneficia más o la utiliza de una manera mayor.

Las conclusiones de este informe, en el que se relaciona el comportamiento de su mercado laboral respecto a la crisis económica actual, se pueden resumir en las siguientes:

\begin{tabular}{|l|l|}
\hline \multicolumn{1}{|c|}{ Zona geográfica } & \multicolumn{1}{c|}{ Personas afectadas } \\
\hline Países nórdicos & $\begin{array}{l}\text { Aquellas personas con trabajo declarado que, no obstante, } \\
\text { han venido complementando sus ingresos con trabajo } \\
\text { oculto, son los que más han sufrido el descenso en el } \\
\text { tamaño de la economía sumergida en la presente crisis. }\end{array}$ \\
\hline Países del sur europeo & $\begin{array}{l}\text { Son las personas desempleadas las que más están su- } \\
\text { friendo el decrecimiento de la economía no declarada. Es } \\
\text { probable que se deba a los efectos más pronunciados de la } \\
\text { crisis sobre determinados sectores económicos. }\end{array}$ \\
\hline
\end{tabular}




\begin{tabular}{|l|l|}
\hline \multicolumn{1}{|c|}{ Zona geográfica } & \multicolumn{1}{c|}{ Personas afectadas } \\
\hline $\begin{array}{l}\text { Países del occidente y } \\
\text { del este europeo }\end{array}$ & $\begin{array}{l}\text { Aunque los desempleados hayan podido acceder a más } \\
\text { trabajos en la economía sumergida, son peor pagados que } \\
\text { aquellos otros trabajadores procedentes de grupos con } \\
\text { ingresos declarados que han accedido a trabajos irregulares. }\end{array}$ \\
\hline
\end{tabular}

Con respecto a las razones que explican esta realidad, este informe de Eurofound entiende que la principal causa se debe a los diferentes grados de economía de bienestar existentes en los países europeos.

Así, en los países que no apuestan por una política laboral activa en beneficio de las clases más desfavorecidas y que presentan menores grados de bienestar social y reducidos pagos compensatorios a los desempleados, las personas suelen recurrir a trabajos no declarados y a la economía sumergida. Por el contrario, en los países donde los beneficios a los desempleados están más extendidos, tanto en relación con colectivos beneficiarios como en cuantías, y son más próximos a los salarios medios, los desempleados no tienen tanta necesidad de acudir a la economía sumergida.

La conclusión es, por tanto, evidente: en cuanto más amplia es la política social, mayor es el impacto en la consecución de la reducción o eliminación de la economía sumergida. ${ }^{19}$

Sin embargo, esta explicación plantea serias dudas si se pretende aplicar a España, donde la tasa de economía subterránea es relativamente elevada, y, en cambio, existen cuantiosas políticas de protección social. Quizá la explicación a este comportamiento se tenga que buscar en la forma de financiación del conjunto del Estado de bienestar, vía impuestos indirectos o mediante la imposición a las rentas del trabajo y del capital.

Asimismo, si se compara el nivel de corrupción de un determinado país y el tamaño de su economía sumergida, se deduce una alta relación entre el nivel de corrupción de cada país con el tamaño de la actividad que se escapa del conocimiento y control del Estado. Así, en los países que muestran mayores niveles de economía sumergida se dan altos índices de corrupción. Las causas son diversas: poca transparencia en el funcionamiento de las instituciones

19 Comisión Europea, Undeclared work in the European Union, ComisiSpecial Eurobarometer 402 / Wave EB79.2-TNS opinion \& social, marzo de 2014. 
públicas; impuestos elevados; alto nivel de gasto público; poca transparencia en la financiación de los partidos políticos; poca transparencia de las normas, leyes y procedimientos; falta de eficientes controles institucionales; excesiva burocracia, y crecimiento de los intercambios internacionales.

Por tanto, la conclusión es relevante: cualquier actuación que se lleve a cabo en una de estas dos magnitudes (corrupción o economía sumergida), o en ambas al mismo tiempo, tiene un efecto directo de descenso o desaparición en la otra.

Finalmente, si se analiza la incidencia de la economía sumergida en los diferentes sectores de la actividad económica, se puede comprobar que aquellos sectores más regulados, que se basan en contratos con un alto nivel de formalidad entre proveedores y clientes, como pueden ser el suministro de electricidad o los servicios financieros, tienen los menores niveles de economía sumergida. Por el contrario, la construcción, el sector manufacturero y las ventas mayoristas y minoristas son los sectores con mayores cifras de economía sumergida, como se puede observar en la siguiente gráfica.

\section{Gráfica 6}

IMPACTO DE LA ECONOMÍA SUMERGIDA POR SECTORES

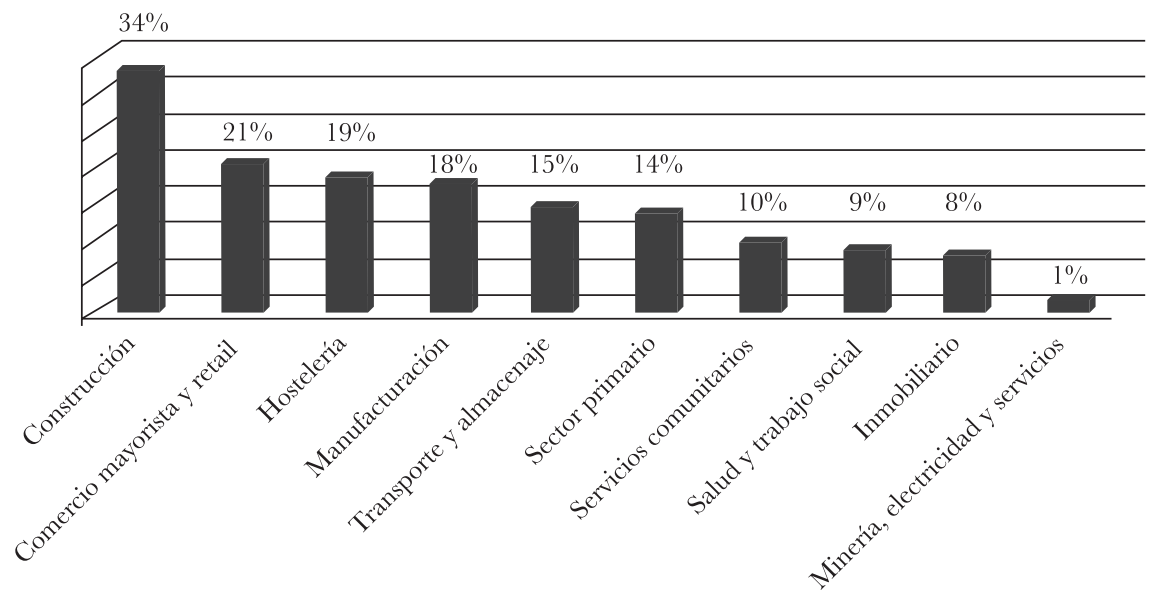

Fuente: Schneider, F. y Kepler, J., Universidad de Linz, Austria; Eurostat, A. T. Kearney Analysis, 2013. 
En concreto, hay que tener en cuenta el relevante efecto que ha tenido en España la crisis inmobiliaria. ${ }^{20} \mathrm{El}$ sector de la construcción en España habría caído un $27 \%$ del valor alcanzado en 2008 , frente a una caída del $12 \%$ en la UE-27, y el número de personas empleadas habría caído un 43\%, frente a un $15 \%$ en la UE-27. Esto podría justificar que la economía sumergida se haya mantenido constante o, incluso, haya caído ligeramente en España en este periodo.

El tamaño de la economía sumergida en España, de acuerdo con las mediciones del informe Schneider referidas a 2013 (18.6\% del PIB), aunque muy próxima a la media europea (18.5\% del PIB), está muy alejada de otros países europeos de nuestro entorno; por ejemplo, Alemania (13\%), Francia (10\%) o Reino Unido (10\%).

Es evidente que, con independencia de la fiabilidad que pueda tener este dato, dado lo difícil que es determinar realmente el peso de la economía sumergida, lo más significativo de los datos manejados es que el nivel de la economía sumergida en España sistemáticamente se ha situado muy por encima de otros Estados europeos, a excepción de Italia.

Además, el impacto de la economía sumergida en España no se da de manera uniforme en todas las comunidades autónomas, ya que en algunas comunidades está más extendida que en otras, tal y como se desprende del informe elaborado por la Asociación de Técnicos del Ministerio de Hacienda (GESTHA), del que se extrae la siguiente tabla.

\section{Tabla 2}

DisTRIBUCIÓN DE LA ECONOMÍA SUMERGIDA POR COMUNIDADES AUTÓNOMAS

\begin{tabular}{|c|c|c|}
\hline Comunidad autónoma & $\begin{array}{c}\text { Porcentaje del PIB } \\
\text { sumergido }\end{array}$ & Total del PIB sumergido \\
\hline Cataluña & $22.3 \%$ & 43.704 \\
\hline Madrid & $19.6 \%$ & 36.556 \\
\hline Andalucía & $24.0 \%$ & 34.182 \\
\hline Comunidad Valenciana & $24.3 \%$ & 24.707 \\
\hline Galicia & $26.3 \%$ & 13.917 \\
\hline
\end{tabular}

20 Schneider, F. y Johannes Kepler, J., "The Shadow Economy...”, cit. 


\begin{tabular}{|c|c|c|}
\hline Comunidad autónoma & $\begin{array}{c}\text { Porcentaje del PIB } \\
\text { sumergido }\end{array}$ & Total del PIB sumergido \\
\hline Castilla y León & $25.0 \%$ & 13.652 \\
\hline País Vasco & $20.6 \%$ & 13.560 \\
\hline Canarias & $28.7 \%$ & 11.563 \\
\hline Castilla-La Mancha & $26.5 \%$ & 9.253 \\
\hline Aragón & $25.5 \%$ & 8.356 \\
\hline Murcia & $24.3 \%$ & 6.546 \\
\hline Baleares & $19.2 \%$ & 5.060 \\
\hline Asturias & $20.2 \%$ & 4.633 \\
\hline Navarra & $20.0 \%$ & 3.575 \\
\hline Extremadura & $19.2 \%$ & 3.474 \\
\hline Cantabria & $23.7 \%$ & 3.206 \\
\hline La Rioja & $31.4 \%$ & 2.432 \\
\hline
\end{tabular}

Fuente: Técnicos del Ministerio de Hacienda (GESTHA) a partir de datos de presión fiscal de Eurostat de 2009. Datos en millones de euros.

Como se puede observar, aunque los peores resultados en términos porcentuales, respecto al tamaño de su economía, se concentraban en La Rioja (31.4\%), Canarias $(28.7 \%)$ y Castilla-La Mancha (26.5\%), hay cuatro comunidades (Cataluña, Madrid, Andalucía y Valencia) que presentaban las mayores cifras en valores absolutos y por sí solas, donde acumulaban más del 58\% del total de la economía sumergida en España.

Sobre la evolución del tamaño de la economía sumergida en España, un estudio de la Fundación de las Cajas de Ahorro realizado en 2011 presenta las siguientes conclusiones: ${ }^{21}$

\begin{tabular}{|l|l|}
\hline 1 & $\begin{array}{l}\text { Se ha producido un crecimiento notable de la economía sumergida en el periodo } \\
1980 / 2008, \text { que se atribuye en gran medida al crecimiento de la carga fiscal. }\end{array}$ \\
\hline 2 & Parece existir una cierta tolerancia hacia estas actividades irregulares. \\
\hline 3 & $\begin{array}{l}\text { En el periodo 1980/2008, el PIB se ha multiplicado por dos y la economía su- } \\
\text { mergida por cuatro, pasando de porcentajes inferiores al 12\% a cercanos al } 24 \% .\end{array}$ \\
\hline 4 & $\begin{array}{l}\text { La economía sumergida es considerada como una válvula de escape en } \\
\text { situaciones de recesión. }\end{array}$ \\
\hline
\end{tabular}

21 Arrazola, M. et al., Dos ensayos de actualidad..., cit. 
En cuanto a las causas de la realidad de la economía sumergida en España, los motivos pueden ser varios:

- En primer lugar, es probable que exista un problema de escasa conciencia fiscal entre los ciudadanos españoles, directamente relacionado con la percepción de que es nula o muy baja la posibilidad de detectar el fraude y, por consiguiente, escasa la actividad sancionatoria; incluso, se podría hablar de la existencia de una doble moral en esta materia.

- En segundo lugar, puede existir la idea de que la regulación actual relacionada con la materia laboral y/o fiscal es excesivamente compleja, especialmente si se aplica a las pymes, por lo que no queda más remedio que incumplirla.

- Finalmente, hay que tener en cuenta que la presión fiscal y en materia de cotizaciones a la seguridad social es una de las más altas en los países de la Unión Europea. ${ }^{22}$

\section{MEDIDAS PARA REDUCIR LA ECONOMÍA SUMERGIDA}

Las medidas adoptadas para reducir la economía sumergida durante la crisis, según el informe de Eurofound, son de dos tipos: disuasorias, que se basan en la detección y penalización del incumplimiento, e incentivadoras, que se centran en favorecer la transferencia de trabajo no declarado hacia el declarado, tratando de incrementar el compromiso social con la economía oficial, que se pueden sintetizar en la siguiente tabla:

Tabla 3

MEDIDAS APLICADAS PARA REDUCIR LA ECONOMÍA SUMERGIDA

\begin{tabular}{|c|c|l|}
\hline Medidas & Tipos & \multicolumn{1}{c|}{ Actuaciones } \\
\hline \multirow{4}{*}{ Disuasorias } & Detección & $\begin{array}{l}\text { Comprobación, intercambio de datos y } \\
\text { cooperación. }\end{array}$ \\
\cline { 2 - 3 } & \multirow{2}{*}{ Sanciones } & Mejora de la efectividad de las inspecciones. \\
\cline { 3 - 3 } & & Aumento de las sanciones por evasión fiscal. \\
\cline { 2 - 3 } & Fuerte penalizaciones por incumplimientos. \\
\hline
\end{tabular}

22 Servicios de Estudios, CEOE, Nota informativa núm. 251, 13/2014, 18 de junio de 2014, bttp:// wnw.ceoe.es/ resources/image/ na251.pdf. 


\begin{tabular}{|c|c|c|c|}
\hline Medidas & Tipos & & Actuaciones \\
\hline \multirow{16}{*}{ Incentivadoras } & \multirow{6}{*}{ Preventivo } & \multicolumn{2}{|c|}{ Simplificación del cumplimiento. } \\
\hline & & \multicolumn{2}{|c|}{ Uso de herramientas tecnológicas. } \\
\hline & & \multicolumn{2}{|c|}{ Incentivos fiscales. } \\
\hline & & \multicolumn{2}{|c|}{ Transición hacia el autoempleo. } \\
\hline & & \multicolumn{2}{|c|}{ Nuevos tipos de trabajo. } \\
\hline & & \multicolumn{2}{|c|}{ Desarrollo de microempresas. } \\
\hline & \multirow{6}{*}{ Curativo } & \multirow{3}{*}{$\begin{array}{l}\text { Incentivos } \\
\text { al comprador }\end{array}$} & Vales de servicios. \\
\hline & & & $\begin{array}{l}\text { Impuestos directos } \\
\text { focalizados. }\end{array}$ \\
\hline & & & $\begin{array}{l}\text { Impuestos indirectos } \\
\text { específicos. }\end{array}$ \\
\hline & & \multirow{3}{*}{$\begin{array}{l}\text { Incentivos } \\
\text { al vendedor }\end{array}$} & Amnistías fiscales. \\
\hline & & & Divulgación voluntaria. \\
\hline & & & Asesoramiento empresarial. \\
\hline & \multirow{4}{*}{$\begin{array}{l}\text { Fomento del } \\
\text { compromiso }\end{array}$} & \multicolumn{2}{|c|}{ Promoción del trabajo declarado. } \\
\hline & & \multicolumn{2}{|l|}{ Educación. } \\
\hline & & \multicolumn{2}{|c|}{ Justicia procesal. } \\
\hline & & \multicolumn{2}{|c|}{ Justicia redistributiva. } \\
\hline
\end{tabular}

Fuente: Williams, C. y Renooy, P., Tackling undeclared work in 27 European Union Member States and Norway: Approaches and measures since 2008, Dublin, Eurofound, 2013.

En este mismo sentido, como se puede apreciar en el siguiente gráfico, en 2001 y 2003 las medidas disuasorias fueron las que comúnmente se aplicaron. Sin embargo, en 2005 un mayor número de países comenzaron a aplicar medidas incentivadoras, principalmente preventivas (por ejemplo, simplificación de exigencias, facilitar la transición hacia trabajo declarado, desarrollo de microempresas, entre otras) y curativas (como incentivos a compradores y proveedores, amnistías, etcétera). 


\section{Gráfica 7}

TIPOS DE MEDIDAS APLICADAS, SEGÚN LOS AÑOS

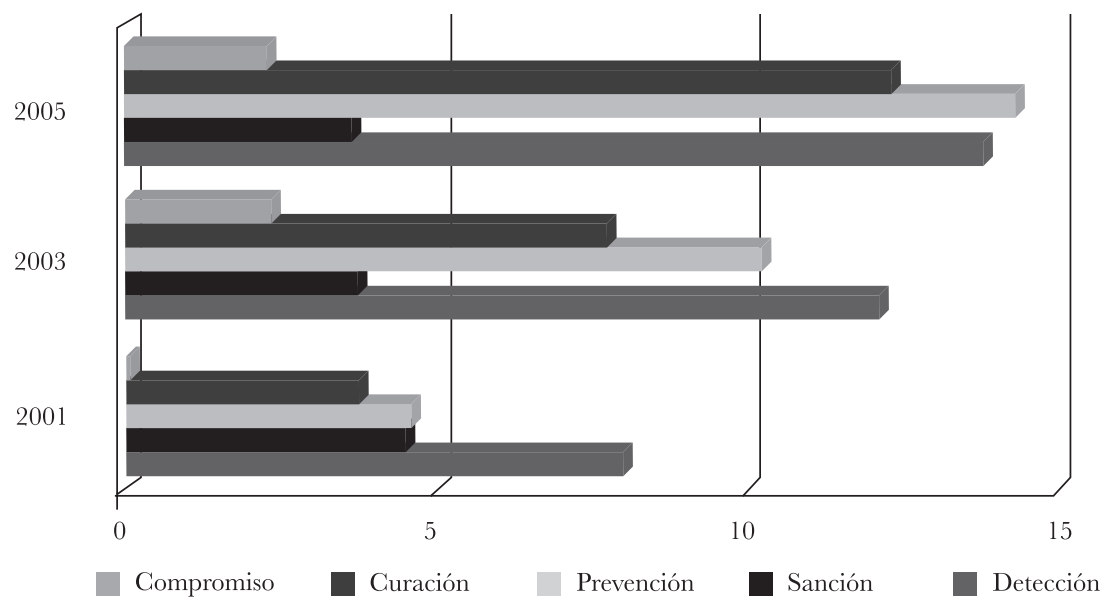

Fuente: Williams, C. y Renooy, P., Tackling undeclared work in 27 European Union Member States and Norway: Approaches and measures since 2008, Dublin, Eurofound, 2013.

Esta tendencia se ha mantenido a partir del inicio de la crisis, si bien se puede observar una ampliación importante en el elenco de medidas aplicadas por los diferentes países de la Unión Europea. De acuerdo con los datos de 2010, la totalidad de los países de la Unión Europea habían reforzado las medidas de detección; un 93\% había incrementado las sanciones; un 90\% había iniciado la adopción de medidas preventivas, y sólo un 64\% había adoptado otras medidas de carácter curativo.

Finalmente, si se comprueba el grado de eficacia de los diferentes tipos de las medidas adoptadas para luchar contra la economía sumergida, se puede observar, como se muestra en la siguiente gráfica, que las medidas disuasorias son las más importantes y que, en el otro extremo, las medidas incentivadoras son, con diferencia, las menos relevantes. 


\section{GRÁFICA 8 \\ GRADO DE RELEVANCIA DE LAS MEDIDAS TOMADAS}

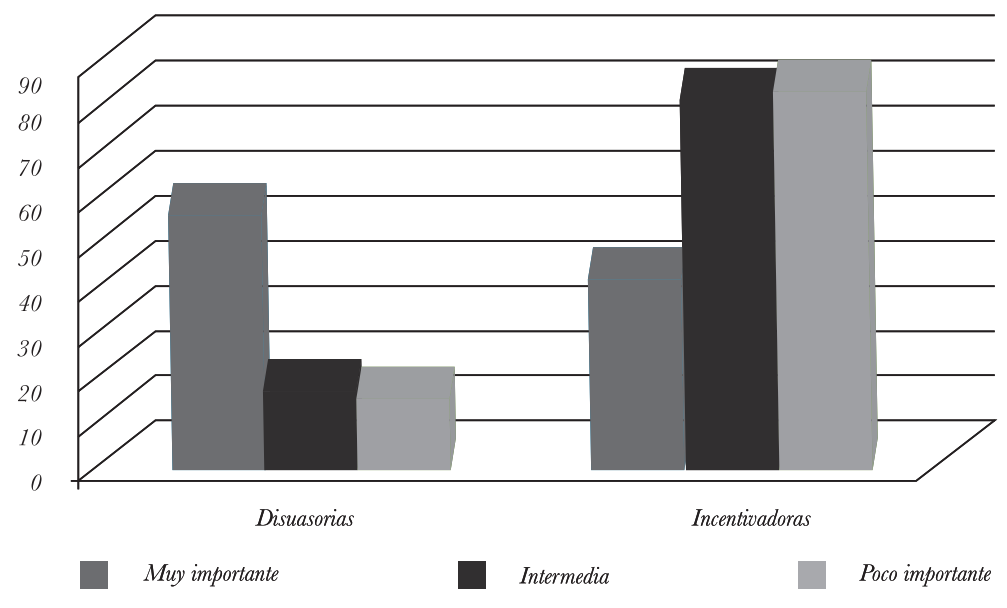

Fuente: Williams, C. y Renooy, P., Tackling undeclared work, in 27 European Union Member States and Norway: Approaches and measures since 2008, Dublin, Eurofound, 2013.

\section{CONCLUSIONES}

Analizada la situación de la economía sumergida y de su inmediata consecuencia tanto en Europa como concretamente en España, que es el fraude, es necesario establecer medidas que permitan que las relaciones laborales vuelvan a situaciones anteriores a las sucesivas crisis económicas y, especialmente, en superación de la actual.

El derecho laboral ha de volver a ser el elemento referencia en el nexo jurídico establecido entre el trabajador y la empresa. Es necesario que desaparezcan amenazas actuales, como la proliferación del trabajo no decente en los trabajadores ${ }^{23}$ o el desarrollo de competencia desleal entre empresas. De su recuperación depende el futuro de la economía de Europa del siglo XXI. ${ }^{24}$

Mientras se consigue la desaparición o, en su caso, la reducción a niveles mínimos de la economía sumergida, se formulan las siguientes propuestas:

23 Véase http:// wnw.ilo.org/global/ about-the-ilo/decent-work-agenda/lang--es/index.htm.

24 La economía informal y el trabajo decente: una guia de recursos sobre politicas, apoyando la transición hacia la formalidad, Ginebra, OIT, 2013. 
- Es necesario establecer medidas con un enfoque global, cuyo objetivo sea la lucha contra el fraude.

Se ha de partir de un diagnóstico que fije la realidad de la situación y de los parámetros en los que se funda, con objeto de que estas medidas sean realmente efectivas.

Estas medidas han de estar suficientemente coordinadas entre los distintos organismos de la administración.

Sería deseable que estas medidas fueran preventivas y orientadas a interrumpir el cumplimiento de la normativa en origen. Estas medidas han de ser evaluadas regularmente.

- Mejora de la eficiencia de los sistemas de control tanto en relación con el cumplimiento de las obligaciones como respecto al uso correcto de los bienes y servicios públicos.

- Desarrollo de estrategias de comprobación, cooperación e intercambio de información, tanto en el ámbito nacional como en el internacional, desde una vertiente administrativa y/o judicial.

- Flexibilización de las cargas y procedimientos administrativos, especialmente para las pymes y los trabajadores autónomos, introduciendo el uso de los medios tecnológicos.

- Simplificación de las figuras impositivas, con una rebaja lineal de los tipos impositivos y la correspondiente eliminación de las bonificaciones a la cotización de la seguridad social.

- Implantación de medidas cuyo objetivo consista en aumentar la sensibilización fiscal de los ciudadanos, y en el caso del ámbito laboral, haciendo especial incidencia en las pymes y en los trabajadores autónomos, con objeto de reforzar la conciencia social para que los contribuyentes no sólo cumplan con sus deberes, sino también para que puedan colaborar con la administración en la erradicación de bolsas de fraude.

- Modificación de la normativa laboral en aquellos aspectos que sean necesarios, haciéndola más adaptable a la realidad existente en las empresas españolas en cada momento y teniendo en cuenta el tejido empresarial.

\section{BIBLIOGRAFÍA}

AibAr BERnAD, J., "La lucha contra el fraude en el ingreso de recursos de la Seguridad Social”, Revista de Trabajo y Seguridad Social (CEF), núm. 351, 2012. 
Arrazola, M. et al., "Estimación del volumen de economía sumergida en España”, Cuadernos de Información Económica, núm. 220, 2011.

- Dos ensayos de actualidad sobre la economía española. La economía sumergida en España, Funcas, 2011.

BILETTA, I. y MEIXNER, M., Industrial relations and undeclared work, European Foundation for the Improvement of Living and Working Conditions, 2005.

CAMINO FRíAS, J., Lucha contra el empleo irregular y el fraude a la seguridad social, Valladolid, Lex Nova, 2013.

CírCUlo DE EMPRESARIOS, Implicaciones de la economía sumergida en España. XXV T Edición del Libro Marrón, Círculo de Empresarios, 2010.

FERNÁNDEZ DíAZ, A., "Corrupción y economía sumergida: una visión de conjunto”, Implicaciones de la economía sumergida en España. XXVI Edición del Libro Marrón, Círculo de Empresarios, 2010.

FERNÁNDEZ ORRICO, F. J., La inspección de trabajo frente al fraude de las prestaciones de la seguridad social, Madrid, Ministerio de Empleo y Seguridad Social, 2012.

Gestha, “Observaciones de Gestha al Anteproyecto de Ley de modificación de la normativa tributaria y presupuestaria y de adecuación de la normativa financiera para la intensificación de las actuaciones de prevención y lucha contra el fraude", 2012.

Gil VillanUEVA, M., "La Ley 13/2012, de 26 de diciembre, de lucha contra el empleo irregular y el fraude a la Seguridad Social", Relaciones Laborales, núm. 2, 2013.

INSTITUTO DE ESTUDIOS FISCALES, Opiniones y actitudes fiscales de los españoles en 2011, documento de trabajo, núm. 19, Madrid, 2012.

ISSA, Seguridad social dinámica: un compromiso global para la excelencia, noviembre de 2013.

JimÉNEZ FERnÁNDEZ, A. y MARTíneZ PARDO-DEL VAlLE, R., La economía sumergida en España, documentos de trabajo, núm. 4, Madrid, Fundación de Estudios Financieros, 2013.

LIZCANO ÁlvAREZ, J., "Corrupción a nivel nacional e internacional: La transparencia como antídoto", Implicaciones de la economía sumergida en España. XXVI Edición del Libro Marrón, Círculo de Empresarios, 2010. 
OrganizACión PROFESIONAL DE INSPECTORES DE HACIENDA DEL ESTADO, "Lucha contra el fraude fiscal en España. Deficiencias y medidas necesarias", 2012.

, "Problemas de la Agencia Tributaria en la lucha contra el fraude fiscal", 2013.

, "XIII Encuentros Tributarios 2013. Propuestas contra la utilización de los paraísos fiscales", 2013.

PFAU-EFFINGER, B., "Varieties of Undeclared Work in European Societies", British Journal of Employment Relations, vol. 41, 1, 2009.

PICKARDT, M. y SARDÁ, J., "Size and causes of the underground economy in Spain: A correction of the record and new evidence from the MCDR approach", 2013.

RiCHARD Murphy, R., "Closing de European Tax Gap”, Tax Research UK.

Rocha SÁnchEZ, F., "El trabajo no declarado en España", Estudios de la Fundación, núm. 45, mayo de 2011.

SCHNEIDER, F., "The Shadow Economy and Shadow Labor Force: A Survey of Recent Developments", IZA Discussion Paper No. 8278, bttp://ssrn. com/abstract $=2462710$.

, "Size and development of the shadow economy from 2003 to 2012: some new facts", 2013.

y JOHANNES KePLER, J., "The Shadow Economy in Europe, 2013”, Informe ATKearney/Visa, 2013.

- et al., Shadow Economies All over the World. New Estimates for 162 Countries from 1999 to 2007, World Bank, 2010.

y Enste, D., "Shadow Economies Around the World: Size, Causes and Consequences", IMF, Working Paper, WP/00/26, Washington DC, 2000 .

SEmpere Navarro, A. V., El trabajo sumergido, Madrid, Editorial Universitaria Ramón Areces, 2011.

SERRANO SANZ, J. M., Desigualdades territoriales en la economía sumergida, Zaragoza, Confederación de Empresarios de Aragón, 2012.

The Tax Justice Network, "The Cost of Tax Abuse. A briefing paper on the cost of tax evasion worldwide", The Tax Justice Network, 2011. 
TOROLlo GONZÁLEZ, F. J., “La Ley 13/2012 y la lucha contra el empleo irregular y el fraude a la seguridad social: antecedentes y contexto normativo", Revista Española de Derecho del Trabajo, núm. 158, 2013.

Williams, C. y ReNoOY, P., Tackling undeclared work in 27 European Union Member States and Norway: Approaches and measures since 2008, Dublin, Eurofound, 2013. Ty 